Artículo

\title{
Botryosphaeriaceae: una familia de hongos, compleja, diversa y cosmopolita
}

\author{
Alejandra Mondragón-Flores ${ }^{1,2}$ \\ Gerardo Rodríguez-Alvarado ${ }^{2}$ \\ Nuria Gómez-Dorantes ${ }^{2}$ \\ Jesús Jaime Guerra-Santos ${ }^{3}$ \\ Sylvia Patricia Fernández-Pavía ${ }^{2 \S}$ \\ ${ }^{1}$ Campo Experimental Valle de Apatzingán-INIFAP. Carretera Apatzingán-Cuatro caminos km 17.5, \\ Antúnez, Michoacán. CP. 60780. Tel. 800 0882222, ext. 84610. (mondragon.flores@ gmail.com). ${ }^{2}$ Instituto \\ de Investigaciones Agropecuarias y Forestales-UMSNH. Carretera Morelia-Zinapécuaro km 9.5, \\ Tarímbaro, Michoacán. CP. 58880. Tel. 443 3223500, ext. 5226. (fernandezpavia@ hotmail.com; \\ gra.labpv@gmail.com; nuriah@live.com.mx). ${ }^{3}$ Universidad Autónoma del Carmen-Facultad de Ciencias \\ Naturales-Centro de Investigación de Ciencias Ambientales. Calle Laguna de Términos s/n, col. Renovación \\ $2^{\text {a }}$ sección, Ciudad del Carmen, Campeche, México. CP. 24155. Tel. 938 1343965. (jjguerra- \\ santos@hotmail.com). \\ ${ }^{\S}$ Autora para correspondencia: fernandezpavia@hotmail.com.
}

\section{Resumen}

En la última década se ha incrementado el interés por estudiar los hongos de la familia Botryosphaeriaceae debido a las enfermedades que inducen en cultivos de importancia económica a su amplia distribución cosmopolita y a la asociación observada entre la patogénesis y el estrés del hospedante. Se han reportado más de diez especies asociadas a síntomas en diferentes partes de una misma planta, lo que indica que un número importante de especies de esta familia no presentan especificidad en rango de hospedantes. Además, diversos estudios han demostrado la capacidad de estos hongos para 'brincar' de sus hospedantes nativos originales a cultivos agrícolas que se establecen en áreas cercanas, pertenecientes a la misma familia botánica o a una familia diferente. El objetivo de este trabajo es revisar marcadores morfológicos y moleculares para la identificación taxonómica de especies de la familia Botryosphaeriace, su distribución geográfica, rango de hospedantes agrícola y aspectos de desarrollo para la enfermedad incluyendo modos de dispersión. La información presentada podrá ser de utilidad en la etiología, identificación y diagnóstico de especies de Botryosphaeriace así como el manejo de las enfermedades causadas que ellas inducen.

Palabras clave: estrés, hospedantes, síntomas.

Recibido: enero de 2021

Aceptado: marzo de 2021 


\section{Introducción}

La familia Botryosphaeriaceae está representada por 23 géneros de hongos cosmopolitas con una amplia gama de hospedantes y que pueden ser endófitos, fitopatógenos o saprófitos (Slippers y Wingfield, 2007; Slippers et al., 2017). Miembros de esta familia inducen enfermedades en plantas que se encuentran bajo estrés (De Wet et al., 2003). Pueden causar síntomas como cancros, gomosis, muerte descendente de ramas, defoliación y necrosis de hojas y muerte de la planta; al conjunto de estos síntomas se le llama síndrome de declinamiento (Slippers y Wingfield, 2007).

En los últimos años, en diversos hospedantes se han descrito especies desconocidas, en nuevas regiones geográficas (Netto et al., 2017). Lo anterior indica que estos hongos se están dispersando por todo el mundo como resultado de la globalización comercial principalmente, convirtiéndolos en una amenaza potencial para los agroecosistemas, bosques, plantas nativas e introducidas (Slippers et al., 2017).

\section{Identificación de las especies de Botryosphaeriaceae}

La familia Botryosphaeriaceae fue introducida por Theissen y Sydow (1918) y pertenece al orden Botryosphaeriales (Schoch et al., 2006). Los géneros con distribución geográfica mundial y afectan a un mayor número de hospedantes son: Diplodia, Dothiorella, Lasiodiplodia y Neofusicoccum, englobando 70\%, con un aproximado de 300 especies fitopatógenas (Slippers et al., 2017).

Las características morfológicas de micelio y cuerpos fructíferos asexuales son utilizadas para la identificación a nivel de género y especie (Alves et al., 2006; Sandoval-Sánchez et al., 2013). Sin embargo, estas características son muy variables y en ocasiones no son distintivas, por que comparten entre especies; además son influenciadas por la edad y el sustrato donde crecen los aislados, por lo tanto, es necesario el uso de herramientas moleculares (Alves et al., 2007).

Las secuencias de los genes que codifican para el factor de elongación 1- $\alpha$ (TEF1- $\alpha)$, beta tubulina $(\beta-T u b)$ y los espaciadores internos transcritos (ITS), son las regiones moleculares más utilizadas en los análisis filogenéticos para la identificación de especies de Botryosphaeriaceae (White et al., 1990; Alves et al., 2006; Zhou et al., 2015). Otro gen que también es útil es la segunda subunidad grande de la ARN polimerasa II (RPB2) (Fernández-Herrera et al., 2017).

Recientemente, Lopes y colaboradores (2017) sugirieron que los genes MAT1-2-1 y MAT1-1-1 son eficientes en la resolución de especies dentro del género Neofusicoccum y fueron útiles para demostrar que la mayoría de las especies son homotálicas. Una desventaja de usar estos marcadores es que no siempre se pueden obtener ambos genes o sólo está disponible un aislado de una especie. El gen MAT1-2-1 es más preciso y confiable para la diferenciación de especies por contener menos intrones conservados y se obtiene una mejor amplificación por PCR.

Otra herramienta para diferenciar especies de Botryosphaeriaceae es el análisis por secuencias internas repetidas simples (ISSR), la cual es una técnica simple, reproducible, rápida y útil cuando se trata de secuenciar una gran cantidad de aislados, agrupar a los de una misma especie, determinar variabilidad interespecífica y diferenciar especies críticas, así como detectar variabilidad intraespecífica (Zhou et al., 2001). 
En la familia Botryosphaeriaceae se han identificado especies híbridas, morfotipos y complejos de especies crípticas que ocupan el mismo nicho ecológico. En estos casos es difícil diferenciar las especies cuando solamente se utiliza uno o dos loci para su identificación (De Wet et al., 2003; Cruywagen et al., 2017). El análisis multilocus de secuencias de ADN y el uso de una mayor cantidad de aislados en los análisis, es actualmente la forma más eficiente de reconocer especies híbridas, morfotipos y complejo de especies crípticas (Cruywagen et al., 2017).

Para la diferenciación eficiente de morfotipos, De Wet et al. (2003) usaron el análisis de seis genes codificantes de proteínas beta tubulina $(\beta-T u b)$, quitina sintetasa $(C H S)$, factor de elongación 1- $\alpha$ (TEF 1- $\alpha)$, actina (ACT), calmodulina (CAL) y glutaraldehído-6-fosfato $(G P D)$, así como en seis loci microsatélites (SS5, SS7, SS8, SS9, SS10 y SS11).

\section{Distribución}

Slippers et al. (2017), Indican que los hongos fitopatógenos pertenecientes a Botryosphaeriaceae afectan generalmente plantas en zonas subtropicales y tropicales; sin embargo, en los últimos años se ha reportado su presencia en el mundo, ejemplo de lo anterior son las especies de Neofusicoccum que se sabe colonizan a 46 hospedantes de 18 familias botánicas en diez países incluyendo todos los continentes (Sakadilis et al., 2011).

Esta capacidad de infectar múltiples hospedantes y migrar entre ellos facilita la propagación de especies y genotipos de Botryosphaeriaceae en nuevas áreas (Mehl et al., 2017). Algunos miembros de esta familia pueden tener cierta especificidad, que está influenciada por el hospedante y su hábitat (Slippers y Wingfield, 2017). Las actividades humanas que influyen en la dispersión de fitopatógenos y sus interacciones con sus hospedantes son: la introducción de plantas no nativas en nuevas áreas, cambios en el uso de la tierra y la deforestación intensiva (Pavlic-Zup et al., 2015).

Además, cuando estos hongos infectan plantas a temperaturas altas y sequía, pueden convertirse en patógenos muy agresivos y resultar en una amenaza potencial para los agroecosistemas, bosques naturales, plantas nativas e introducidas (Piskur et al., 2011). Lo anterior ha ocasionado un incremento en el interés por estudiar estos hongos debido a su presencia en múltiples hospedantes, nuevas áreas geográficas y a su agresividad en hospedantes bajo estrés (Slippers et al., 2017).

\section{Rango de hospedantes}

Las especies fitopatógenas de Botryosphaeriaceae atacan plantas leñosas (Sakalidis et al., 2011). Se considera que atacan principalmente angiospermas, aunque en el caso de las especies de Diplodia las cuales están restringidas a gimnospermas, se ha sugerido que proviene de un ancestro de Botryosphaeriaceae que evolucionó en las angiospermas (De Wet et al., 2008). Existen estudios que demuestran la capacidad de estos hongos para moverse de hospedantes nativos a no nativos y entre plantas cercanas o lejanas filogenéticamente (Sakadilis et al., 2013; Pavlic-Zup et al., 2015).

Algunas de las nuevas especies que han sido identificadas están restringidas a un solo hospedante y son poco patogénicas, lo que sugiere que son de reciente introducción o que sólo están asociadas de manera endofítica (Pérez et al., 2010). Sin embargo, se han reportado casos de hospedantes infectados por solo una o por más de diez especies asociadas a síntomas en diferentes partes de la planta, indicando que pueden infectar indistintamente a más de un órgano (Delgado-Cerrone et al., 2016; Mayorquin et al., 2016; Tedihou et al., 2017). 
Algunos autores indican que la especie más frecuentemente aislada en un hospedante usualmente no es la más agresiva (Mayorquin et al., 2016). Debido a la falta de consistencia para delimitar las especies de Botryiosphaeria, es difícil cuantificar cuantas plantas atacan, sin embargo, en un estudio reciente se determinó un aproximado de 1692 hospedantes en el mundo (Batista et al., 2012). Estos patógenos atacan a plantas forestales ornamentales, y diversos hospedantes agrícolas, incluyendo árboles frutales tropicales (Fernández-Herrera et al., 2017; Lawrence et al., 2017) (Cuadro 1), arbustos caducifolios, herbáceas y palmas (Cuadro 2).

Cuadro 1. Distribución de especies de Botryosphaeriaceae, asociados a cancro, gomosis, declinamiento y pudriciones en árboles frutales perennes.

\begin{tabular}{|c|c|c|c|}
\hline Hospedante & Especies & País & Referencia \\
\hline $\begin{array}{l}\text { Citrus } \mathrm{x} \\
\text { cinensis }\end{array}$ & $\begin{array}{l}\text { Lasiodiplodia theobromae, } \\
\text { Neoscytalidium dimidiatum }\end{array}$ & $\begin{array}{l}\text { Italia, } \\
\text { México }\end{array}$ & $\begin{array}{l}\text { Polizzi et al. (2009); } \\
\text { Polanco-Florián et al. (2019) }\end{array}$ \\
\hline $\begin{array}{l}\text { Citrus } \\
\text { latifolia }\end{array}$ & $\begin{array}{l}\text { Lasiodiplodia citricola, Lasiodiplodia } \\
\text { iraniensis, L. pseudotheobromae, L. } \\
\text { theobromae, Lasiodiplodia subglobosa, } \\
\text { L. citricola. }\end{array}$ & México & $\begin{array}{l}\text { Bautista-Cruz et al. (2018); } \\
\text { Valle-De la Paz et al. (2019) }\end{array}$ \\
\hline Citrus x limon & $\begin{array}{l}\text { L. pseudotheobromae, L. theobromae, } \\
\text { Neofusicoccum australe, } \\
\text { Neofusicoccum parvum, Neoscytalidium } \\
\text { hyalinum, Spencermartinsia viticola }\end{array}$ & $\begin{array}{l}\text { Brasil, EE. } \\
\text { UU, Turquía }\end{array}$ & $\begin{array}{l}\text { Adesemoye y Eskalen } \\
\text { (2011); Mayorquin } \text { et al. } \\
\text { (2012); Awan et al. }(2016) \text {; } \\
\text { Guajardo } \text { et al. (2018) }\end{array}$ \\
\hline Citrus spp. & $\begin{array}{l}\text { Diplodia mutila, Diplodia seriata, } \\
\text { Dothiorella viticola, Diplodia iberica, } \\
\text { L. citricola*, Lasiodiplodia } \\
\text { hormozganensis, Lasiodiplodia } \\
\text { iraniensis L. theobromae, Lasiodiplodia } \\
\text { parva, N. australe, Neofusicoccum } \\
\text { dimidiatum, Neofusicoccum luteum, } \\
\text { Neofusicoccum mediterraneum, } N . \\
\text { parvum. }\end{array}$ & $\begin{array}{l}\text { EE. UU, } \\
\text { Emiratos } \\
\text { Árabes } \\
\text { Unidos, Irán, } \\
\text { Oman }\end{array}$ & $\begin{array}{l}\text { Abdollahzadeh } \text { et al. (2010); } \\
\text { Al-Sadi et al. (2013); } \\
\text { Adesemoye } \text { et al. (2014) }\end{array}$ \\
\hline $\begin{array}{l}\text { Dimocarpus } \\
\text { longan }\end{array}$ & $\begin{array}{l}\text { L. hormozganensis, L. iraniensis, L. } \\
\text { pseudotheobromae, L. theobromae }\end{array}$ & Puerto Rico & Serrato-Díaz et al. (2019) \\
\hline $\begin{array}{l}\text { Mangifera } \\
\text { indica }\end{array}$ & $\begin{array}{l}\text { Botryosphaeria fabicerciana, Diplodia } \\
\text { allocellula, Lasiodiplodia brasiliense, } \\
\text { Lasiodiplodia crassispora, } \\
\text { Lasiodiplodia gonubiensis, } \\
\text { Lasiodiplodia egyptiacae, L. } \\
\text { hormozganensis }{ }^{*} \text {, L. iraniensis, } \\
\text { Lasiodiplodia mahajangana L. } \\
\text { pseudotheobromae, L. theobromae, } \\
\text { Lasiodiplodia viticola, N. } \\
\text { mediterraneum, N. parvum, } \\
\text { Neofusicoccum umdonicola, } \\
\text { Neofusicoccum vitifusiforme, } \\
\text { Pseudofusicoccum olivaceum }\end{array}$ & $\begin{array}{l}\text { Irán, Egipto, } \\
\text { Emiratos } \\
\text { Arabis } \\
\text { Unidos, } \\
\text { México, } \\
\text { Perú, } \\
\text { Sudáfrica, } \\
\text { Tailandia }\end{array}$ & $\begin{array}{l}\text { Abdollahzadeh et al. (2010); } \\
\text { Ismail et al. (2012); Al-Sadi } \\
\text { et al. }(2013) ; \text { Sandoval- } \\
\text { Sánchez et al. (2013); } \\
\text { Trakunyingcharoen } \text { et al. } \\
\text { (2014); Mehl } \text { et al. (2017) }\end{array}$ \\
\hline
\end{tabular}




\begin{tabular}{|c|c|c|c|}
\hline Hospedante & Especies & País & Referencia \\
\hline $\begin{array}{l}\text { Nephelium } \\
\text { lappaceum }\end{array}$ & $\begin{array}{l}\text { L. brasiliensis, L. hormozganensis, L. } \\
\text { iraniensis, L. pseudotheobromae, L. } \\
\text { theobromae, Neofusicoccum } \\
\text { batangarum, N. parvum }\end{array}$ & Puerto Rico & Serrato-Díaz et al. (2019) \\
\hline $\begin{array}{l}\text { Persea } \\
\text { americana }\end{array}$ & $\begin{array}{l}\text { D. mutila, D. seriata, Dothiorella } \\
\text { iberica, Fusicoccum aesculi, L. } \\
\text { theobromae, } N \text {. australe, } N . \text { luteum, } \\
\text { Neofusicoccum nonquaesitum, } N . \\
\text { parvum, Neofusicoccum sp. }\end{array}$ & $\begin{array}{l}\text { Chile, EE. } \\
\text { UU, México }\end{array}$ & $\begin{array}{l}\text { McDonald et al. (2011); } \\
\text { Molina-Gayosso et al. } \\
\text { (2012); Valencia et al. } \\
\text { (2019) }\end{array}$ \\
\hline $\begin{array}{l}\text { Pouteria } \\
\text { sapota }\end{array}$ & L. theobromae & México & Tovar-Pedraza et al. (2012) \\
\hline
\end{tabular}

Cuadro 2. Distribución de especies de Botryosphaeriaceae, asociados a cancros, gomosis, declinamiento y pudriciones en hospedantes arbustivos, caducifolios, herbáceas y palmas.

\begin{tabular}{|c|c|c|c|}
\hline Hospedante & Especies & País & Referencia \\
\hline $\begin{array}{l}\text { Anacardium } \\
\text { occidental }\end{array}$ & $\begin{array}{l}\text { Lasiodiplodia brasiliense, } \\
\text { Lasiodiplodia euphorbicola, } \\
\text { Lasiodiplodia gonubiensis, L. } \\
\text { iraniensis, Lasiodiplodia } \\
\text { jatrophicola, Lasiodiplodia } \\
\text { gravistriata*, L. pseudotheobromae, } \\
\text { L. theobromae, Neofusicoccum } \\
\text { batangarum, Pseudofusicoccum } \\
\text { stromaticum }\end{array}$ & Brasil & Netto et al. (2017) \\
\hline $\begin{array}{l}\text { Actinidia } \\
\text { chinensis }\end{array}$ & $\begin{array}{l}\text { Botryosphaeria dothidea, N. parvum, } \\
\text { L. theobromae }\end{array}$ & China & Zhou et al. (2015) \\
\hline Carica papaya & $\begin{array}{l}\text { L. brasiliense }{ }^{*} \text { L. hormozganensis, } \\
\text { Lasiodiplodia marypalme* }, \text { L. } \\
\text { pseudotheobromae, L. theobromae. }\end{array}$ & Brasil & Netto et al. (2014) \\
\hline Cocos nucifera & $\begin{array}{l}\text { L. brasiliense, L. egyptiacae, L. } \\
\text { pseudotheobromae, L. theobromae }\end{array}$ & Brasil, China & $\begin{array}{l}\text { Rosado et al. (2016); Zhang y } \\
\text { Niu (2019) }\end{array}$ \\
\hline $\begin{array}{l}\text { Fragaria } \mathrm{x} \\
\text { ananassa }\end{array}$ & Macrophomina phaseolina & $\begin{array}{l}\text { Chile, } \\
\text { España, } \\
\text { República de } \\
\text { Tunecina }\end{array}$ & $\begin{array}{l}\text { Avilés et al. (2008); Sánchez } \\
\text { et al. (2013); Hajlaoui et al. } \\
\text { (2015) }\end{array}$ \\
\hline $\begin{array}{l}\text { Malus } \\
\text { domestica }\end{array}$ & $\begin{array}{l}\text { B. dothidea, Diplodia intermedia, D. } \\
\text { seriata, Diplodia pseudoseriata, } L . \\
\text { pseudotheobromae, L. theobromae, } \\
\text { N. australe, N. luteum, N. parvum }\end{array}$ & $\begin{array}{l}\text { China, } \\
\text { Uruguay }\end{array}$ & $\begin{array}{l}\text { Delgado-Cerrone et al. } \\
\text { (2016); Xue et al. (2019) }\end{array}$ \\
\hline
\end{tabular}




\begin{tabular}{|c|c|c|c|}
\hline Hospedante & Especies & País & Referencia \\
\hline Olea sp. & L. hormozganensis ${ }^{*}$ & Irán & Abdollahzadeh et al. (2010) \\
\hline Prunus persica & $\begin{array}{l}\text { B. dothidea, D. seriata, } D . \\
\text { intermedia, } N . \text { parvum, } N \text {. luteum, D. } \\
\text { pseudoseriata, N. australe, } L . \\
\text { theobromae }\end{array}$ & China & $\begin{array}{l}\text { Tian et al. (2018); Wang et al. } \\
\text { (2011) }\end{array}$ \\
\hline $\begin{array}{l}\text { Pyrus } \\
\text { communis }\end{array}$ & $\begin{array}{l}\text { B. dothidea, Botryosphaeria rhodina, } \\
\text { Botryosphaeria obtusa, B. parva }\end{array}$ & China & Zhai et al. (2014) \\
\hline Rubus idaeus & Neofusicoccum algeriense & México & Serret-López et al. (2017) \\
\hline $\begin{array}{l}\text { Rubus } \\
\text { subgénero } \\
\text { Eubatu }\end{array}$ & L. theobromae, L. parva & México & Contreras-Pérez et al. (2019) \\
\hline $\begin{array}{l}\text { Saccharum } \\
\text { officinarum }\end{array}$ & M. phaseolina & México & Leyva-Mir et al. (2015) \\
\hline Vaccinium spp. & $\begin{array}{l}\text { B. dothidea, L. theobromae, } N . \\
\text { parvum, } N \text {. australe, Neofusicoccum } \\
\text { eucalyptorum }\end{array}$ & $\begin{array}{l}\text { China, } \\
\text { México, } \\
\text { Portugal }\end{array}$ & $\begin{array}{l}\text { Mondragón-Flores et al. } \\
\text { (2012); Xu et al. (2015); } \\
\text { Boyzo-Marín et al. (2016); } \\
\text { Hilário et al. (2019) }\end{array}$ \\
\hline Vitis vinifera & $\begin{array}{l}\text { D. seriata, D. mutilata, N. australe, } \\
\text { N. luteum, N. parvum }\end{array}$ & $\begin{array}{l}\text { Nueva } \\
\text { Zelanda }\end{array}$ & $\begin{array}{l}\text { Billones-Baaijens et al. } \\
\text { (2015) }\end{array}$ \\
\hline
\end{tabular}

* = especies descritas recientemente.

\section{Factores que condicionan la enfermedad}

Las especies fitopatógenas de Botyosphaeriaceae tienen una gran diversidad de hospedantes, son hongos oportunistas capaces de colonizar un gran número de especies botánicas y causar enfermedad en aquellas que se encuentran bajo algún tipo de estrés, principalmente hídrico (De Wet et al., 2003). Lo anterior podría explicarse porque en respuesta a la falta de agua, la planta aumenta los niveles de ácido abscísico y se reprimen sus defensas reguladas por ácido jasmónico, etileno y ácido salicílico y como consecuencia la susceptibilidad a patógenos se incrementa (Asselbergh et al., 2008).

Esto es importante ante las condiciones emergentes de cambio climático que no solo aumenta el estrés en las comunidades vegetales, sino que también favorece el desarrollo de patógenos y sus tasas de supervivencia (Slippers y Wingfield, 2017). El estrés en las plantas modifica su susceptibilidad a patógenos lo que provoca cambios en el impacto de las enfermedades en los cultivos (Elad y Pertot, 2014). 


\section{Síntomas}

Enfermedades de consideración en diversos cultivos de importancia agrícola están asociadas a miembros de Botryosphaeriaceae (Eskalen et al., 2013; Bautista-Cruz et al., 2018). Algunos estudios hechos por Rosado et al. (2016), indican que con frecuencia múltiples especies del mismo género, así como de diferentes géneros, están asociadas con los síntomas y es difícil diferenciarlos para cada especie (Delgado-Cerrone et al., 2016).

En algunos casos ha sido posible asociar síntomas con la especie del patógeno debido a ciertas características como la presencia de anillos en las lesiones (Tian et al., 2018). Los síntomas asociados a estos hongos son: cancro en ramas y tronco, declinamiento, gomosis, muerte descendente de ramas, necrosis en hojas, pudriciones de semillas, frutos, pedúnculo, raíz y tizón de brotes e inflorescencias (Slippers et al., 2005; Sandoval-Sánchez et al., 2013; Dugan et al., 2015; Hajlaoui et al., 2015; Netto et al., 2017; Rodríguez-Gálvez et al., 2017).

\section{Trasmisión y dispersión}

De acuerdo con Bihon et al. (2011), estos hongos se trasmiten de manera horizontal de plantas maduras a plantas jóvenes por medio de esporas; sin embargo, la trasmisión vertical aún no ha sido comprobada. La liberación de las esporas es frecuente durante el período de lluvias que en otras estaciones y estas se dispersan por salpicaduras de agua de lluvia (Skalen et al., 2013).

Investigaciones indican que además de su potencial como patógenos, pueden asociarse a hongos de otras familias y ser trasmitidos por el escarabajo de la corteza (Hypocryphalus mangiferae), que actúa como vector en la dispersión entre los hospedantes (Adawi et al., 2006). Por otra parte, se cree que la muerte descendente causada por estos hongos podría servir como atenuante de otras enfermedades más importantes como la muerte repentina del roble, al matar ramas que de otra manera producirían hojas que se infectan fácilmente con Phytophthora ramorum y que se diseminan por salpicaduras de agua de lluvia (Lawrence et al., 2017).

Cuando los frutales son sometidos a prácticas culturales, las heridas de poda son una fuente de estrés para la planta y proporcionan un punto de entrada a los patógenos que colonizan los tejidos de forma basipetal (Rodríguez-Gálvez et al., 2017). Existen reportes que indican que la presencia de una herida no es necesaria para que los hongos infecten los órganos del hospedante, sin embargo, sí contribuyen a aumentar la gravedad de los síntomas (Zhou et al., 2015).

Factores abióticos como el incremento de la temperatura, humedad relativa alta, sequías, heladas, altas densidades de siembra y malas prácticas de poda, favorecen el desarrollo de la enfermedad aumentando la incidencia que oscila de 20 a $97 \%$ en algunos cultivos (De la Mora-Castañeda $e t$ al., 2014; Fernández-Herrera et al., 2017; Bautista-Cruz et al., 2018).

\section{Conclusiones}

La familia Botryosphaeriaceae es de gran importancia por las enfermedades que ocasionan en cultivos de importancia agrícola en todas las áreas templadas, tropicales y subtropicales en todo el mundo. Su habilidad para pasar de endófito a patógeno en plantas bajo estrés, representa una amenaza en cultivos sometidos a condiciones subóptimas de desarrollo, estrés hídrico, por ejemplo. 
Debido a la falta de información en aspectos de reproducción, supervivencia, dispersión y técnicas de detección e identificación precisas, a nivel mundial se requieren más estudios de estos patógenos, para establecer medidas de control y manejo de las enfermedades que ocasionan.

\section{Literatura citada}

Abdollahzadeh, J.; Javadi, A.; Goltapeh, M. E.; Zare, R. and Phillips, A. J. L. 2010. Phylogeny and morphology of four new species of Lasiodiplodia from Iran. Persoonia. 25(10):1-10. Doi: $10.3767 / 003158510 \times 524150$.

Adesemoye, A. O. and Eskalen, A. 2011. First report of Spencermartinsia viticola, Neofusicoccum australe and N. parvum causing branch canker of citrus in California. Plant Dis. 95(6):770. https://doi.org/10.1094/PDIS-02-11-0092.

Adesemoye, A. O.; Mayorquin, J. S.; Wang, D. H.; Twizeyimana, M.; Lynch, S. C. and Eskalen, A. 2014. Identification of species of Botryosphaeriaceae causing bot gummosis in citrus in California. Plant disease. 98(1):55-61. Doi: 10.1094/PDIS-05-13-0492-RE.

Al-Adawi, A. O.; Deadman, M. L.; Al-Rawahi, A. K.; Al-Maqbali, Y. M.; Al-Jahwari, A. A.; AlSaadi, B. A.; Al-Amri, I. S. and Wingfield, M. J. 2006. Etiology and causal agents of mango sudden decline disease in the Sultanate of Oman. Eur. J. Plant Pathol. 116(4):247-254. Doi: 10.1007/s10658-006-9056-X.

Al-Sadi, A. M.; Al-Wehaibi, A. N.; Al-Shariqi, R. M.; Al-Hammadi, M. S.; Al-Hosni, I. A.; AlMahmooli, I. H. and Al-Ghaithi, A. G. 2013. Population genetic analysis reveals diversity in Lasiodiplodia species infecting date palm, citrus, and mango in oman and the UAE. Plant Disc. 97(10):1363-1369. Doi: 10.1094/PDIS-03-13-0245-RE.

Alves, A.; Correia, A. and Phillips, A. J. L. 2006. Multi-gene genealogies and morphological data support Diplodia cupressi sp. nov., previously recognized as D. pinea f. sp. cupressi, as a distinct species. Fungal Diversity. 23(1):1-15.

Alves, A.; Phillips, A. J. L.; Henriques, I. and Correia, A. 2007. Rapid differentiation of species of botryosphaeriaceae by PCR fingerprinting. Res. Microbiol. 158(2):112-121. https://doi.org/10.1016/j.resmic.2006.10.003.

Asselbergh, B.; De-Vleesschauwer, D. and Höfte, M. 2008. Global switches and fine- tuning ABA modulates plant pathogen defense. Mol. Plant Microbe Interact. 21(6):709-719. Doi: 10.1094/MPMI-21-6-0709.

Avilés, M.; Castillo, S.; Bascon, J.; Zea Bonilla, T.; Martín-Sánchez, P. M. and Pérez-Jiménez, R. M. 2008. First report of Macrophomina phaseolina causing crown and root rot of strawberry in Spain. Plant Pathol. 57(2):382-382. Doi: 10.1111/j.1365-3059.2007.01717.x.

Awan, Q. N.; Akgül, D. S. and Unal, G. 2016. First Report of Lasiodiplodia pseudotheobromae causing postharvest fruit rot of lemon in Turkey. Plant Disc. 100(11):2327. Doi: 10.1094/PDIS-04-16-0512-PDN.

Batista, E.; Lopes, A. and Alves, A. 2012. What do we know about Botryosphaeriaceae? an overview of a worldwide cured dataset. Forests. 12(3):313. https://doi.org/10.3390/ f12030313.

Bautista-Cruz, M. A.; Almaguer-Vargas, G.; Leyva-Mir, S. G.; Colinas-León, M. T.; Correia, K. C.; Camacho-Tapia, M.; Robles-Yerena, L.; Michereff, S. J. and Tovar-Pedraza, J. M. 2018. Phylogeny, distribution, and pathogenicity of Lasiodiplodia species associated with cankers and dieback symptoms of persian lime in Mexico. Plant Dis. 103(6):1156-1165. Doi: 10.1094/PDIS-06-18-1036-RE. 
Billones-Baaijens, R.; Ridgway, H. J.; Jones, E. E. and Jaspers, M. V. 2015. Spatial distribution of Neofusicoccum species within a rootstock mother vine indicates potential infection pathways. Eur. J. Plant Pathol. 141(2):267-279. Doi: 10.1007/s10658-014-0540-4.

Bihon, W.; Slippers, B.; Burgues, T.; Wingfield, M. J. and Wingfield, B. D. 2011. Sources of Diplodia pinea endophytic infections in Pinus patula and $P$. radiata seedlings in South Africa. Forest Pathol. 41(5):370-375. Doi: 10.1111/j.1439-0329.2010.00691.x.

Boyzo-Marin, J.; Rebollar-Alviter, A.; Silva-Rojas, H. V. and Ramirez-Maldonaldo, G. 2016. First report of Neofusicoccum parvum causing stem blight and dieback of blueberry in Mexico. Plant Dis. 100(12):2524-2524. https://doi.org/10.1094/PDIS-02-17-0251-PDN.

Contreras-Pérez, M.; Santoyo-Pizano, G.; De los Santos-Villalobos, S.; Gutiérrez-García, M. A.; Orozco-Mosqueda, M. C. and Rocha-Granados, M. C. 2019. First report of Lasiodiplodia on blackberry plants (Rubus subgenus Eubatus) in the Michoacan state, Mexico. Mex. J. Phytopathol. 37(3):479-485. Doi: 10.18781/R.MEX.FIT.1905-4.

Cruywagen, E. M.; Slippers, B.; Roux, J. and Wingfield, M. J. 2017. Phylogenetic species recognition and hybridisation in Lasiodiplodia: a case study on species from baobabs. Fungal Biol. 121(4):420-436. Doi: 10.1016/j.funbio.2016.07.014.

Delgado-Cerrone, L.; Mondino-Hintz, P. and Alaniz-Ferro, S. 2016. Botryosphariaceae species associated with stem canker, die-back and fruit rot on apple in Uruguay. Eur. J. Plant Pathol. 146(3):637-655. Doi: 10.1007/s10658-016-0949-z.

De-Wet, J.; Burguess, T.; Slippers, B.; Preisig, O.; Wingfield, B. D. and Wingfield, M. J. 2003. Multiple gene genealogies and microsatellite markers reflect relationships between morphotypes of Sphaeropsis sapinea and distinguish a new species of Diplodia. Mycol. Res. 107(5):557-566. https://doi.org/10.1017/S0953756203007706.

De-Wet, J.; Slippers, B.; Preisig, O.; Wingfield, B. D. and Wingfield, M. J. 2008. Phylogeny of the Botryosphaeriaceae reveals patterns of host association. Mol. Phylogen. Evol. 46(1):116126. Doi: 10.1016/j.ympev.2007.08.016.

Dugan, F. M.; Lupien, S. L.; Osuagwu, A. N.; Uyoh, E. A.; Okpako, E. and Kisha, T. 2015. New records of Lasiodiplodia theobromae in seed of Tetrapleurra tetraptera form Nigeria and fruit of Cocos nucifera from Mexico. J. Phytopathol. 164(1):65-68. Doi: 10.1111/JPH.12384.

Eland, Y. and Pertot, I. 2014. Climate change impacts on plant pathogens and plant diseases. J. Crop Improv. 28(1):99-139. Doi: 10.1080/15427528.2014.865412.

Eskalen, A.; Faber, B. and Bianchi, M. 2013. Spore trapping and pathogenicity of fungi in the Botryosphaeriaceae and Diaporthaceae associated with avocado branch canker in California. Plant Dis. 97(3):329-332. Doi: 10.1094/PDIS-03-12-0260-RE.

Fernández-Herrera, E.; Moreno-Salazar, S.; Rentería-Martínez, M. E.; Arratia-Castro, A. A. and Villar-Luna, E. 2017. Neoscytalidium dimidiatum: causal agent of dieback in Ficus benjamina L. in Mexico. Rev. Chapingo. Ser. Hortic. 23(3):203-210. Doi: 10.5154/r.rchsh.2017.02.009.

Guajardo, J.; Riquelme, N.; Tapia, L.; Larach, A.; Torres, C.; Camps, R. and Besoain, X. 2018. First report of Lasiodiplodia theobromae causing bot gummosis in Citrus limon in Chile. Plant Dis. 102(4):818. DOI: 10.1094/PDIS-09-17-1517-PDN.

Hajlaoui, M. R.; Mnari-Hattab, M.; Sayen, M.; Zarrouk, I.; Jemmali, A. and Koike, S. T. 2015. First report of Macrophomina phaseolina causing charcoal rot of strawberry in Tunisia. New Dis. Rep. 32(1):14. Doi: 10.5197/j.2044-0588.2015.032.014.

Hilário, S.; Lopes, A.; Santos, L. and Alves, A. 2019. Botryosphaeriaceae species associated with blueberry stem blight and dieback in the centre region of portugal. Eur. J. Plant Pathol. 156(3):31-44. Doi: 10.1007/s10658-019-01860-6. 
Ismail, A. M.; Cirvilleri, G.; Polizzi, G.; Crous, P. W.; Groenewald, J. Z. and Lombard, L. 2012. Lasiodiplodia species associated with dieback disease of mango (Mangifera indica) in egypt. Australasian Plant Pathol. 41(6):649-660. https://doi.org/10.1007/s13313-012-0163-1.

Lawrence, P.; Peduto, H. F.; Gubler, W. D. and Trouillas, F. P. 2017. Botryosphaeriaceae species associated with dieback and canker disease of bay laurel in northern California with the description of Dothiorella californica sp. nov. Fungal Biol. 121(4):347-360. Doi: 10.1016/j.funbio.2016.09.005.

Leyva-Mir, S. G.; Velázquez-Martínez, G. C.; Tlapal-Bolaños, B.; Tovar-Pedraza J. M.; RosasSalto, G. H. y Alvarado-Gómez, O. G. 2015. Caracterización morfológica y molecular de aislados de Macrophomina phaseolina asociados a caña de azúcar en México. Rev. Argentina Microbiol. 47(2):143-147. https://doi.org/10.1016/j.ram.2015.03.003.

Lopes, A.; Phillips, A. J. L. and Alves, A. 2017. Mating type genes in the genus Neofusicoccum: Mating strategies and usefulness in species delimitation. Fungal Biol. 121(4):394-404. Doi: 10.1016/j.funbio.2016.08.011.

Mayorquin, J. S.; Wang, D. H.; Twizeyimana, M. and Eskalen, A. 2016. Identification, distribution, and pathogenicity of Diatrypaceae and Botryosphaeriaceae associated with citrus branch canker in the southern California desert. Plant Dis. 100(12):2402-2413. Doi: 10.1094/PDIS-03-16-0362-RE.

Mc-Donald, V. and Eskalen, A. 2011. Botryosphaeriaceae species associated with avocado branch canker in California. Plant Dis. 95(11):1465-1473. Doi: 10.1094/PDIS-02-11-0136.

Mehl, J. W. M.; Slippers, B.; Roux, J. and Wingfield, M. J. 2017. Overlap of latent pathogens in the Botryosphaeriaceae on a native and agricultural host. Fungal Biol. 121(4):405-419. Doi: 10.1016/j.funbio.2016.07.015.

Molina-Gayosso, E.; Silva-Rojas, H. V.; García-Morales, S. and Ávila-Quezada, G. 2012. First report of black spots on avocado fruit caused by Neofusicoccum parvum in Mexico. Plant Dis. 96(2):287. Doi: 10.1094/PDIS-08-11-0699.

Modragón-Flores, A.; López-Medina, J.; Ochoa-Ascencio, S. y Gutiérrez-Contreras, M. 2012. Hongos asociados a la parte aérea del arándano en Los Reyes, Michoacán, México. Rev. Mex. Fitopatol. 30(2):141-144.

Netto, M. S. B.; Assunção, I. P.; Lima, G. S. A.; Marques, M. W.; Lima, W. G.; Monteiro, J. H. A.; Balbino, V. Q.; Michereff, S. J.; Phillips, A. J. L. and Câmara, M. P. S. 2014. Species of Lasiodiplodia associated with papaya stem-end rot in Brazil. Fungal Divers. 67(1):127141. Doi: 10.1007/s13225-014-0279-4.

Netto, M. S. B.; Lima, W. G.; Correia, K. C.; Da Silva, CH. F. B.; Thon, M.; Martins, R. B.; Miller, R. N. G.; Michereff, S. J. and Camara, M. P. S. 2017. Analysis of phylogeny, distribution, and pathogenicity of Botryosphaeriaceae species associated with gummosis of anacardium in Brazil, with a new species of Lasiodiplodia. Fungal Biol. 121(4):437-451. Doi: 10.1016/j.funbio.2016.07.006.

Pavlic-Zup, D.; Wingfield, M. J.; Boissin, E. and Slippers, B. 2015. The distribution of genetic diversity in the Neofusicoccum parvum/N. ribis complex suggests structure correlated with level of disturbance. Fungal Ecol. 13(13):93-102. Doi: 10.1016/j.funeco.2014.09.002.

Pérez, C. A.; Wingfield, M. J.; Slippers, B.; Altier, N. A. and Blanchette, R. A. 2010. Endophytic and canker-associated Botryosphaeriaceae occurring on non-native eucalyptus and native myrtaceae trees in Uruguay. Fungal Divers. 41(1):53-69. Doi: 10.1007/s13225-009-0014-8.

Pis-kur, B.; Pavlic, D.; Slippers, B.; Ogris, N.; Maresi, G.; Wingfield, M. J. and Jurc, D. 2011. Diversity and pathogenicity of Botryosphaeriaceae on declining Ostrya carpinifolia in Slovenia and Italy following extreme weather conditions. Eur. J. Forest Res. 130(2):235249. Doi: 10.1007/s10342-010-0424-x. 
Polanco-Florián, L. G.; Alvarado-Gómez, O. G.; Pérez-González, O.; González-Garza, R. y Olivares-Sáenz, E. 2019. Hongos asociados con la muerte regresiva de los cítricos en Nuevo León y Tamaulipas, México. Rev. Mex. Cienc. Agríc. 10(4):757-764. Doi: 10.29312/remexca.v10i4.1417.

Polizzi, G.; Aiello, D.; Vitale, A.; Giuffrida, F.; Groenewald, J. Z. and Crous P. W. 2009. First report of shoot blight, canker, and gummosis caused by Neoscytalidium dimidiatum on citrus in Italy. Plant Dis. 93(11):1215-1215. Doi: 10.1094/PDIS-93-11-1215A.

Rodríguez-Gálvez, E.; Guerrero, P.; Barradas, C.; Crous, P. W. and Alves, A. 2017. Phylogeny and pathogenicity of Lasiodiplodia species associated with dieback of mango in Peru. Fungal Biol. 121(4):452-465. Doi: 10.1016/j.funbio.2016.06.004.

Sakalidis, L. M.; Hardy, G. E. S. J. and Burgess, T. I. 2011. Class III endophytes, clandestine movement amongst hosts and habitats and their potential for disease; a focus on Neofusicoccum austral. Australasian Plant Pathol. 40(5):510-521. Https://doi.org/10.1007/s13313-011-0077-3.

Sakalidis, M. L.; Slippers, B.; Wingfield, B. D.; Hardy, G. E. S. J. and Burgess T. I. 2013. The challenge of understanding the origin, pathways and extent of fungal invasions: global populations of the Neofusicoccum parvum/N. ribis species complex. Divers. Distrib. 19(8):873-1094. Doi: 10.1111/DDI.12030.

Sánchez, S.; Gambardella, M.; Henríquez, J. L. and Díaz I. 2013. First report of crown rot of strawberry caused by Macrophomina phaseolina in Chile. Plant Dis. 97(7):996. Doi: 10.1094/PDIS-12-12-1121-PDN.

Sandoval-Sánchez, M.; Nieto-Ángel, D.; Sandoval-Islas, J.; Téliz-Ortiz, D.; Orozco-Santos, M. y Silva-Rojas, H. V. 2013. Hongos asociados a pudrición del pedúnculo y muerte descendente del mango (Mangifera indica L.). Agrociencia. 47(1):61-73.

Schoch, C. L.; Shoemaker, R. A.; Seifert, K. A.; Hambleton, S.; Spatafora, J. W. and Crous, P. W. 2006. A multigene phylogeny of the dothideomycetes using four nuclear loci. Mycologia. 98(6):1041-1052. Doi: 10.3852/mycologia.98.6.1041.

Serret-López, R. E.; Tlapal-Bolaños, B.; Leyva-Mir, S. G.; Correia, K. C.; Camacho-Tapia, M.; Méndez-Jaimes, F. and Tovar-Pedraza, J. M. 2017. First report of Neofusicoccum algeriense causing dieback of red raspberry in Mexico. Plant Dis. 101(9):1673-1673. https://doi.org/10.1094/PDIS-02-17-0251-PDN.

Serrato-Diaz, L. M.; Aviles-Noriega, A.; Soto-Bauzo, A.; Rivera-Vargas, L. I.; Goenaga, R. and Bayman, P. 2019. Botryosphaeriaceae fungi as causal agents of dieback and corky bark in rambutan and Longan. Plant Dis. 104(1):105-115. Doi: 10.1094/PDIS-02-19-0295-RE.

Slippers, B.; Crous, P. W.; Jami F.; Groenewald, J. Z. and Wingfield, M. J. 2017. Diversity in the botryosphaeriales: looking back, looking forward. Fungal Biol. 121(4):307-321. Doi: 10.1016/j.funbio.2017.02.002.

Slippers, B.; Johnson, G. I.; Crous, P. W.; Coutinho, T. A.; Wingfield, B. D. and Wingfield, M. J. 2005. Phylogenetic and morphological re-evaluation of the Botryosphaeria species causing diseases of Mangifera indica. Mycologia. 97(1):99-110. Doi: 10.3852/mycologia.97.1.99.

Slippers, B. and Wingfield, M. J. 2007. Botryosphaeriaceae as endophytes and latent pathogens of woody plants: diversity, ecology and their impact. Fungal Biol. Rev. 21(2-3):90-106. Doi: 10.1016/j.fbr.2007.06.002.

Tedihou, E.; Kpemoua, K. and Tounou, A. 2017. Dépérissement des manguiers et citrus dans la région centrale du togo et méthodes de lutte par des fongicides. J. Appl. Bioscience. 119(1):11829-11838. Doi: 10.4314/jab.v119i1.1.

Theissen, F. and Sydow, H. 1918. Vorentwürfe zu den pseudosphaeriales. Annales Mycol. 16(12):1-34. 
Tian, Y.; Zhao, Y.; Sun, T. and Wang, L. 2018. Identification and characterization of Phomopsis amygdali and Botryosphaeria dothidea associated with peach shoot blight in yangshan, China. Plant Dis. 102(12):2511-2518. Doi: 10.1094/PDIS-02-18-0243-RE.

Tovar-Pedraza, J. M.; Mora-Aguilera, J. A.; Nava-Díaz, C.; Téliz-Ortíz, D.; Valdovinos-Ponce, G.; Villegas-Monter, Á. y Hernández-Morales, J. 2012. Identificación, patogenicidad e histopatología de Lasiodiplodia theobromae en injertos de zapote mamey en Guerrero, México. Agrociencia. 46(2):147-161.

Trakunyingcharoen, T.; Cheewangkoon, R.; To-anun, C.; Crous, P. W.; Van Niekerk, J. M. and Lombard, L. 2014. Botryosphaeriaceae associated with diseases of mango (Mangifera indica). Australasian Plant Pathol. 43(4):425-438. Doi: 10.1007/s13313-014-0284-9.

Valencia, A. L.; Gil, P. M.; Latorre, B. A. and Rosales, I. M. 2019. Characterization and pathogenicity of Botryosphaeriaceae species obtained from avocado trees with branch canker and dieback and from avocado fruit with stem end rot in Chile. Plant Dis. 103(5):996-1005. Doi: 10.1094/PDIS-07-18-1131-RE.

Valle-De la Paz, M.; Guillén-Sánchez, D.; Perales-Rosas, D.; López-Martínez, V.; Juárez-López, P.; Martínez-Fernández, E.; Hernández-Arenas, M.; Ariza-Flores, R. and Gijón-Hernán, A. R. 2019. Distribution, incidence and severity of dieback (Lasiodiplodia spp.) in persa lime in Morelos, Mexico. Mex. J. Phytopathol. 37(3):464-478. Doi: 10.18781/R.MEX.FIT. 1904-7.

Wang, F.; Zhao, L.; Li, G.; Huang, J. and Hsiang, T. 2011. Identification and characterization of Botryosphaeria spp. causing gummosis of peach trees in Hubei province, central China. Plant Dis. 95(11):1378-1384. Doi: 10.1094/PDIS-12-10-0893.

Xu, Ch.; Zhang, H.; Zhou, Z.; Hu, T.; Wang, S.; Wang, Y. and Cao, K. 2015. Identification and distribution of Botryosphaeriaceae species associated with blueberry stem blight in China. Eur. J. Plant Pathol. 143(4):737-752. Doi: 10.1007/s10658-015-0724-6.

Xue, D.; Meng, L.; Li, G.; Li, B. and Wang, C. 2019. First report of Lasiodiplodia pseudotheobromae causing canker and shoot dieback on apple in China. Plant Dis. 103(9):2478. https://doi.org/10.1094/PDIS-01-19-0182-PDN.

Zhang, W. and Niu, X. L. 2019. First report of Lasiodiplodia theobromae causing postharvest stem end rot on coconut in China. Plant Dis. 103(6):1420-1420. https://doi.org/10.1094/PDIS10-18-1861-PDN.

Zhai, L.; Zhang, M.; Lv, G.; Chen, X.; Jia, N.; Hong, N. and Wang, G. 2014. Biological and molecular characterization of four Botryosphaeria species isolated from pear plants showing stem wart and stem canker in China. Plant Dis. 98(6):716-726. Doi: 10.1094/PDIS-10-13-1060-RE.

Zhou, Y.; Gong, G.; Cui, Y.; Zhang, D.; Chang, X.; Hu, R.; Liu, N. and Sun, X. 2015. Identification of Botryosphaeriaceae species causing kiwifruit rot in Sichuan province, China. Plant Dis. 99(5):699-708. https://doi.org/10.1094/PDIS-07-14-0727-RE.

Zhou, S.; Smith, D. R. and Stanosz, G. R. 2001. Differentiation of Botryosphaeria species and related anamorphic fungi using inter simple or short sequence repeat (ISSR) fingerprinting. Mycol. Res. 105(8):919-926. https://doi.org/10.1016/S0953-7562(08)61947-4. 